\title{
Middle Ear Effusion in Mechanically Ventilated Patients: Effects of the Nasogastric Tube
}

\author{
Míriam González Pena MD PhD, Enric Figuerola Massana MD PhD, \\ Pilar Hernández Gutiérrez, and Jordi Rello Condomines MD PhD
}

\begin{abstract}
BACKGROUND: Middle ear effusion (MEE) is rare among adults, but has a higher incidence among ICU patients. The aim of this study was to analyze the effect of nasogastric tube (NGT) on MEE and to assess other predisposing factors. METHODS: Prospective observational study, carrying out an otoscopic examination and tympanometry in $\mathbf{1 0 0}$ mechanically ventilated patients. Immittance testing was carried out within 24 hours of ICU admission and every 72 hours until ICU discharge. In a case of persisting pathologic curve at the moment of discharge from ICU, there was a follow-up examination every 3 days until middle ear function was restored. In addition to descriptive variables, we recorded placement (left or right nostril) and diameter (12, 16, or 18 French) of the NGT. A Cox regression analysis was performed, adjusted for the days since ICU admission. RESULTS: A total of $\mathbf{5 3 5}$ tympanometry studies were carried out, of which 352 were normal and 183 observations presented MEE. We observed that 12 and 16 French NGTs were not significantly associated with abnormal middle ear function, whereas 18 French NGT was significantly associated with MEE (odds ratio $2.54,95 \%$ CI $1.42-4.55 ; P=.01$ ). Other variables independently associated with pathological tympanogram curves were Ramsay Sedation Scale score $\geq 4$ (odds ratio 2.42, 95\% CI 1.65-3.55; $P=.01$ ) and orotracheal intubation (odds ratio 5.72, 95\% CI 3.40-9.60; $P=.01$ ). No intracranial infection or long-term disabilities were identified. CONCLUSIONS: MEEs and tympanometric alterations are frequent in intubated patients (32\% in our study). To prevent these complications, they should receive NGTs with a diameter lower than 18 French, when feasible. Key words: ICU patients; middle ear effusion; nasogastric tube; orotracheal intubation; tympanometry; unconsciousness. [Respir Care 2013;58(2):273-278. (c) 2013 Daedalus Enterprises]
\end{abstract}

\section{Introduction}

Middle ear effusion (MEE) usually manifests as aural fullness and might cause conductive hearing loss. In addition, middle ear fluid will aggravate preexisting sensorineural hearing loss caused by age and can limit the patient's ability to understand commands or explanations. ${ }^{1,2}$

Drs González Pena and Figuerola Massana are affiliated with the Department of Otorhinolaryngology and Head and Neck Surgery, Hospital Universitari de Tarragona Joan XXIII; and Ms Hernández Gutiérrez is affiliated with the Department of Statistics and Epidemiology, L'Institut d'Investigació Sanitària Pere Virgili, Universitat Rovira i Virgili, Tarragona, Spain. Dr Rello Condomines is affiliated with the Intensive Care Unit, Hospital Vall d'Hebron, Centro de Investigación Biomédica en Red de Enfermedades Respiratorias, Universitat Autonoma de Barcelona, Barcelona, Spain.

The authors have disclosed no conflicts of interest.
On the other hand, short and long-term disabilities (acute otitis media, intracranial infection, sepsis, mastoiditis, chronic otitis media), although rare, can result in an untreated otitis media with effusion, especially in patients with immunocompromised state. 3,4

Otitis media with effusion is rare among adults, but its incidence is higher in ICU patients $(25-43.3 \%)$. The middle ear is particularly susceptible to the development of effusion and infection in critically ill patients, due to pre-

\footnotetext{
Correspondence: Míriam González Pena MD PhD, Department of Otorhinolaryngology and Head and Neck Surgery, Hospital Universitari de Tarragona Joan XXIII, L'Institut d'Investigació Sanitària Pere Virgili, Universitat Rovira i Virgili, Tarragona, Spain. E-mail: mgonzalezpena@ gmail.com.
}

DOI: $10.4187 /$ respcare.01911 


\section{Middle Ear Effusion in Mechanically Ventilated Patients}

disposing factors existing in these patients: presence of transnasal tubes, prolonged endotracheal intubation, unconsciousness, alterations in mucociliary function and mucous viscosity, neuromotor dysfunction due to sedation or underlying neurological disease, and, lastly, colonization and migration of nosocomial Gram-negative microorganisms that commonly colonize the oro- and nasopharynx, and adhere to nasogastric and endotracheal tubes. ${ }^{5,6}$

The primary objective of this study was to identify complications in the middle ear associated with mechanical ventilation in the ICU. Secondary objectives were to analyze the role of the nasogastric tube (NGT) in MEE in critically ill patients, and other predisposing factors such as orotracheal intubation and level of unconscious, or tracheostomy. Our hypothesis was that the presence of MEEs in intubated ICU patients might be related to an inability of the Eustachian tube to equalize pressure, due to the effect of nasogastric and orotracheal tubes and due to unconsciousness.

\section{Methods}

A pilot prospective study was conducted from January 2005 through June 2005 with 25 enrolled subjects. Later the study was continued from March 2009 through April 2010. The pilot study comprised the research project for the pre-doctoral studies of the primary author, and the second study was part of her doctoral thesis at Universitat Rovira i Virgili. The institutional ethics board approved the study, and all subjects (or the subject's family in case of unconsciousness) signed an informed consent. A total of 100 critically ill subjects from ICU at Hospital Universitari Joan XXIII in Tarragona, Spain, were included in this study. Subjects included were at least 18 years of age and intubated through the orotracheal route for at least 3 days. Patients excluded were those with a history of ear diseases or intracranial hypertension, ${ }^{7}$ corticosteroid treatment, or nasal packing. All subjects (ear by ear) of this study had to have a normal tympanometry at some point in the study. It was a necessary condition to reject progressive previous causes of Eustachian tube dysfunction (eg, tumor, hypertrophy of adenoid). Data collected later from medical charts were demographic data (age and sex), any underlying diseases of the subjects, duration of orotracheal intubation, level of consciousness, placement and diameter of NGT, and outcome. The Ramsay Sedation Scale (RSS) was used to evaluate the level of consciousness in the subjects. Subjects with scores of $4-6$ on the RSS were considered unconscious. Information regarding the site of the NGTs was collected as we analyzed them ear-by-ear, and the variable was the presence of an NGT only in the ear that was lateralized on the same side as the NGT.

In order to detect MEEs, each subject received both otoscopic examination and tympanometry within 24 hours

\section{QUICK LOOK}

\section{Current knowledge}

Otitis media with effusion is rare among adults, but its incidence is higher in ICU patients. Risk factors for otitis media in mechanically ventilated patients include endotracheal intubation, sedation, presence of gastric tubes, and colonization. The contribution of each of these factors is not well described.

\section{What this paper contributes to our knowledge}

The presence of a nasogastric tube of $\geq 18$ French is an easily modifiable predisposing factor for middle ear effusion in ICU patients. Using the smallest possible diameter for nasogastric tubes may prevent or minimize the effects of middle ear effusion.

of ICU admission, then every 72 hours until ICU discharge. In a case of a persisting pathological curve at the moment of discharge from ICU, there was a follow-up examination every 3 days until middle ear function was restored. The same expert carried out all ear examinations, using a conventional hand-held otoscope. Color and lucency of the tympani and presence of effusions in the middle ear were recorded. Following otoscopic examination and cleaning of the external canal cerumen, an impedance audiometry was carried out, whenever possible, using a portable audiometer (Audiotest 425, Interacoustics, Assens, Denmark). In some cases, it was not possible to carry out a bilateral ear examination because of complications such as clotted blood, cerumen impactation, or fluid drainage on the external ear canal, or because of patient position, cervical collar, head dressings, or lack of subject cooperation. We defined MEE on the basis of tympanometry only, but a previous otoscopy was necessary in order to avoid false positive results of the tympanometry due to cerumen, perforation of the tympani, or inadequate position of the tympanometer probe in the ear canal.

After the otoscopy and tympanometry, we diagnosed MEE depending on the values of pressure and compliance resulting from the tympanometry. For statistical purposes we created the concept of a pathological or normal tympanometric curve. We considered a curve pathological only if both compliance $(<0.35 \mathrm{~mL})$ and pressure $(<-100 \mathrm{daPa})$ were pathological, corresponding to type $\mathrm{B}$ and type $\mathrm{C} 2$ of the Jerger classification. ${ }^{8}$ Subsequently we considered a curve normal that was graded as type $\mathrm{A}$ and type $\mathrm{C} 1$ of the Jerger classification. Our classification aimed to increase statistical strength and to be more restrictive with the concept of a pathological curve (ie, in types $\mathrm{B}$ and type $\mathrm{C} 2$ Jerger curves we were able to find effusions in the middle ear). Furthermore, we found that the concept of the 
Table 1. Subjects' Underlying Diseases

\begin{tabular}{lc}
\hline \hline & $\begin{array}{c}\text { No. of } \\
\text { Subjects } \\
(n=100)\end{array}$ \\
\hline $\begin{array}{l}\text { Respiratory: pneumonia, respiratory failure, } \\
\text { obstructive pulmonary disease, asthma, }\end{array}$ & 22 \\
$\quad$ hemoptysis & \\
Polytrauma & 16 \\
$\begin{array}{l}\text { Neurological: cerebrovascular accident, traumatic } \\
\text { brain injury, meningitis }\end{array}$ & 15 \\
$\begin{array}{l}\text { Abdominal: peritonitis, gastrointestinal bleeding, } \\
\text { mesenteric ischemia, rectal bleeding, pancreatitis }\end{array}$ & 14 \\
$\begin{array}{l}\text { Post-surgery: abdominal aneurysm, } \\
\text { cholecystectomy, intestinal surgery, liver cyst, }\end{array}$ & 14 \\
$\quad$ maxillofacial surgery & 13 \\
$\begin{array}{l}\text { Shock: septic, hypovolemic, and cardiogenic shock } \\
\text { Cardiovascular: ischemia heart diseases, coronary }\end{array}$ & 5 \\
$\quad$ angioplasty & \\
\hline Hepatic encephalopathy & 1 \\
\hline
\end{tabular}

pathological curve correlated better with the pathological otoscopy.

Quantitative variables were described with mean $\pm \mathrm{SD}$, and categorical variables with absolute and relative frequencies. Statistical analysis of pathological curves was carried out by ear, regardless of which subject it belonged to. Kaplan-Meier curves and a log-rank test were used to compare subjects exposed or not to the predisposing factors of MEE (orotracheal intubation, unconsciousness, and NGT) and who presented or not with pathological curve during a determinate time. In order to evaluate the association between pathological curve and the predisposing factors for MEE, we carried out a Cox regression analysis ("Enter" method) adjusted for days from admission. Based on reviewed literature, we introduced the 3 variables that our study was focused on (orotracheal tube, NGT, and unconsciousness). These variables were presented as predisposing factors to developing MEEs in ICU patients. ${ }^{5,6,9,10}$

Results were presented as odds ratios with their respective $95 \%$ confidence intervals. The level of statistical significance was set at $P \leq .05$. Data were analyzed using statistics software (SPSS 15.0, SPSS, Chicago, Illinois).

\section{Results}

Our study comprised 100 subjects, 32 (32\%) of whom were women and $68(68 \%)$ were men, with a mean \pm SD age of $58.1 \pm 17.4$ years. The mean stay in ICU was $10.7 \pm 9.3$ days (range $1-52 \mathrm{~d}$ ). The mean duration of orotracheal intubation until tracheostomy or extubation was $14.5 \pm 5.3$ days, with a median of 16 days. Regarding the clinical evolution of subjects, 17 (17\%) subjects died, while $83(83 \%)$ had a positive outcome. Data on the subjects' underlying diseases are shown in Table 1.

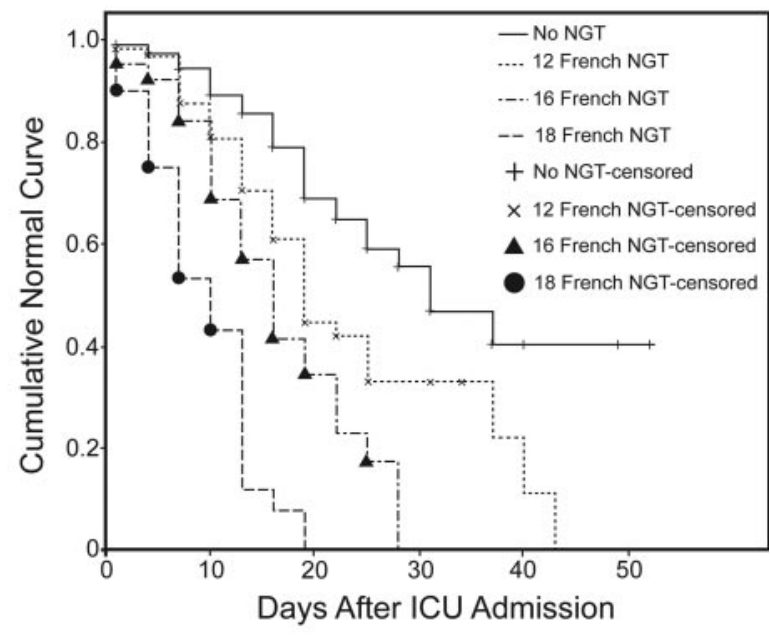

$\begin{array}{lcccccc}\begin{array}{l}\text { Number at } \\ \text { risk }\end{array} & 0 & 10 & 20 & 30 & 40 & 50 \\ & & & & & & \\ \text { No NGT } & 128 & 92 & 33 & 13 & 5 & 2 \\ \begin{array}{l}\text { 12 Fr NGT 202 } \\ \text { 16 Fr NGT } 133\end{array} & 92 & 17 & 8 & 2 & 0 \\ \text { 18 Fr NGT } 72 & 21 & 12 & 0 & 0 & 0 \\ \end{array}$

Fig. 1. Kaplan-Meier curves for pathological curve at different diameters of nasogastric tube (NGT). Fr = French

At the time of admission to ICU, 78 (78\%) subjects had an RSS score within 4-6, whereas $22(22 \%)$ were aware (RSS score 1-3); all subjects (100\%) were intubated through the orotracheal route; $90(90 \%)$ subjects had an NGT, in whom $36(40 \%)$ NGTs were placed in the right nostril and $54(60 \%)$ NGTs in the left nostril, and 7 (7\%) subjects had an orogastric tube and only $3(3 \%)$ subjects did not have any tube. According to the diameter of the NGT, we recorded $43(47.8 \%)$ NGTs with a diameter of 12 French, 32 (35.6\%) NGTs with a diameter of 16 French, and $15(16.7 \%)$ NGTs with a diameter of 18 French. This was carried out by the attending nurse and was not standardized.

We evaluated subjects a total of 380 times. Theoretically, the maximum number of tympanometries we were able to carry out was 760 , and in this study there were 535 tympanometries carried out, and 225 were missing observations due to previously mentioned complications. We obtained a $65 \%$ statistical power with a $95 \%$ confidence level for the 535 real observations. This statistical power could have reached $80 \%$ if there had been no missing observations.

According to the otoscopy we found 343 normal ears and 192 pathologic ears. These results were similar to results obtained from tympanometry: 352 normal ears and 183 pathologic ears. The distribution of pathological tympanometries $(n=183)$ with abnormal compliance and pressure is found in different Kaplan-Meier curves, depending on the predisposing factor for MEE (Figs. 1, 2, and 3). In these graphics, every time that a pathological 


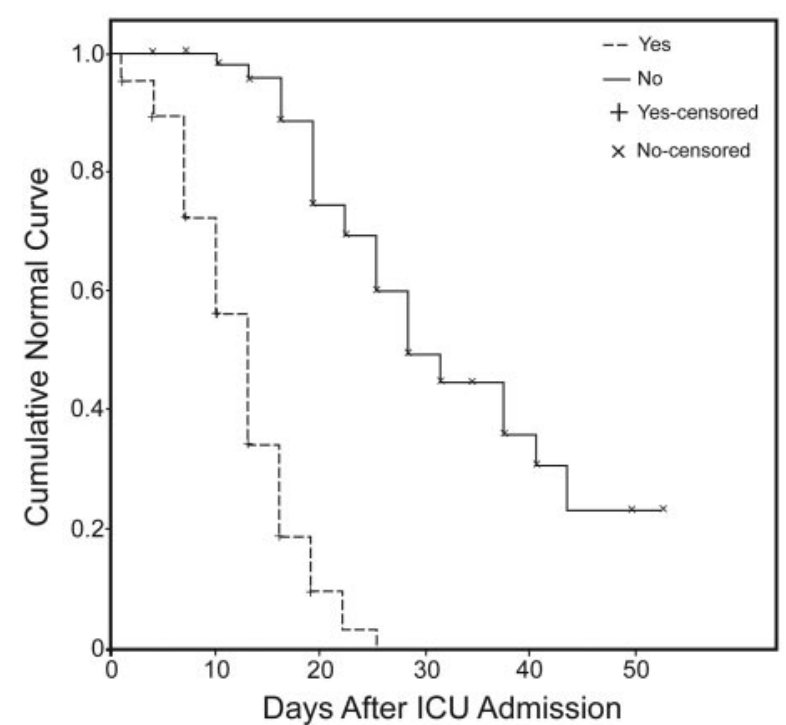

$\begin{array}{ccccccc}\begin{array}{c}\text { Number at } \\ \text { risk }\end{array} & 0 & 10 & 20 & 30 & 40 & 50 \\ \text { Yes } & 360 & 103 & 4 & 0 & 0 & 0 \\ \text { No } & 175 & 157 & 58 & 21 & 7 & 2\end{array}$

Fig. 2. Kaplan-Meier curves for pathological curves, depending on the route of the orotracheal intubation.

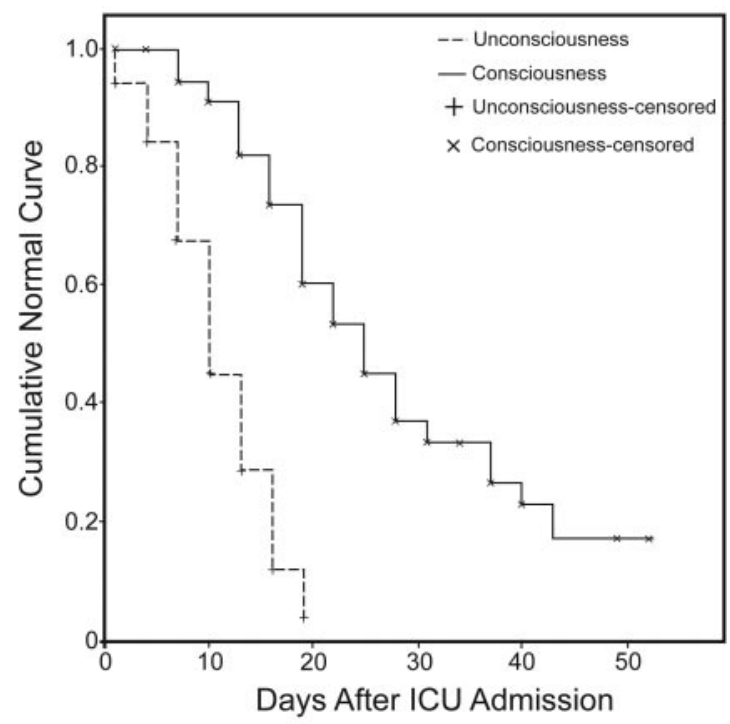

$\begin{array}{lcccccc}\text { Number at risk } & 0 & 10 & 20 & 30 & 40 & 50 \\ \text { Unconsiousness } & 242 & 57 & 0 & 0 & 0 & 0 \\ \text { Consiousness } & 293 & 203 & 62 & 21 & 7 & 2\end{array}$

Fig. 3. Kaplan-Meier curves for pathological curves depending on the level of consciousness.

curve occurred, there was a decrease in the Kaplan-Meier figure. Measures in subjects who had not developed the pathological curve were considered censored observations.
Table 2. Cox Regression Analysis for Pathological Curve Adjusted for ICU Days From Admission

\begin{tabular}{lccc}
\hline \hline $\begin{array}{l}\text { Predisposing Factor for } \\
\text { Middle Ear Effusion }\end{array}$ & $\begin{array}{c}\text { Odds } \\
\text { Ratio* }\end{array}$ & $95 \%$ CI & $P$ \\
\hline $\begin{array}{l}\text { Unconsciousness } \\
\text { Orotracheal intubation }\end{array}$ & 2.42 & $1.65-3.55$ & .01 \\
Nasogastric Tube & 5.72 & $3.40-9.60$ & .01 \\
$\quad$ 12 French & 0.95 & $0.58-1.57$ & .20 \\
16 French & 1.03 & $0.60-1.77$ & .20 \\
18 French & 2.54 & $1.42-4.55$ & .01
\end{tabular}

* Odds ratio of pathologic curve depending on predisposing factors for middle ear effusion.

A Cox regression analysis (Table 2) was used to determine independent predisposing factors for MEE. The variables we entered were: the presence of an orotracheal tube, the presence of an NGT $(12,16$, or 18 French), and unconsciousness. This analysis was adjusted for duration in days from admission to ICU, because degrees of Eustachian tube dysfunction can differ significantly, depending on the exposure time to predisposing factors to MEE. Moreover, a Cox regression analysis revealed an independent association between unconsciousness and orotracheal intubation. Being intubated through the orotracheal route would therefore seem to indicate a variable degree of Eustachian tube dysfunction, without a necessarily low level of consciousness (RSS score 4-6).

At the time of the tracheostomy (26 subjects) or extubation (74 subjects), we reached the maximum of pathological curves, and we found 32 subjects (32\%) with MEE and $68(68 \%)$ subjects who had normal curve of tympanometry. There were 41 pathologic ears in total, with 9 subjects with bilateral MEE and 23 subjects with unilateral MEE. Seventeen subjects died from their underlying diseases, and we followed-up 83 subjects after removing their orotracheal tube. Six days after extubation/tracheostomy, we recorded 11 subjects with MEE (13\%) and 72 subjects $(87 \%)$ who had normal curve. Nine days later, 8 subjects presented MEE (10\%) and 75 subjects $(90 \%)$ had a normal middle ear function.

We found no acute otitis media, intracranial infection, or long-term disabilities in critically ill subjects in this study. However, it was necessary to treat 3 subjects who had carried 18 French NGT, because MEE persisted a month after discharge from ICU. We carried out 2 unilateral myringotomies in 2 subjects, and treated 1 subject with intranasal corticosteroids. Finally, all subjects were able to restore their middle ear function.

\section{Discussion}

Several reports in the literature have analyzed predisposing factors for MEE in ICU patients. ${ }^{5,6,9,10} \mathrm{We}$ have reviewed them and compared them with our own results. 


\section{Middle Ear Effusion in Mechanically Ventilated Patients}

Regarding the NGT, we have found reports that showed NGT as a predisposing factor for MEE, 5,6 but others ${ }^{9,10}$ that could not incriminate NGT as a risk factor for otitis media with effusion. We think that might be due to different effects of NGTs, depending on their diameter. This study is unique in evaluating the effects of NGT in middle ear function of ICU patients, depending on its diameter. In our study, Kaplan-Meier curves (see Fig. 1) showed that subjects who had NGT with a diameter of 18 French presented a higher number of pathological curves, and they appeared sooner than in those subjects who had NGTs of 16 French or 12 French. There were also differences between subjects with or without NGT. Subjects who did not have an NGT presented a lower number of pathological curves, and they occurred later than those subjects with an NGT. A Cox regression analysis revealed a statistically significant association between requiring an 18 French NGT and presenting a pathological curve (odds ratio 2.54, 95\% CI 1.42-4.55). Nasal tubes may impair Eustachian tube function by causing local edema and by obstructing the Eustachian tube ostia. On the other hand, an NGT can interfere with nasopharyngeal clearance, and microorganisms that accumulate in the nasopharynx and adhere to the NGT can seed directly through the Eustachian tube into the middle ear.

In Kaplan-Meier curves for orotracheal intubation (see Fig. 2) we noted that ears of subjects that remained intubated presented a higher number of pathological curves, and they appeared sooner than in those ears of extubated subjects. A Cox regression analysis revealed a statistically significant association between being intubated through the orotracheal route and presenting a pathological curve (odds ratio 5.72, 95\% CI 3.40-9.60). This agrees with reports of other authors. Intubated patients have increased $\mathrm{pH}$ values in the upper airway secretions, which can alter the viscosity of mucus and impair mucociliary clearance. ${ }^{11}$ Furthermore, an orotracheal tube can irritate the oropharyngeal mucosa and cause deterioration in the aeration of upper airway.

In this study, in agreement with other reports in the literature, we found that unconscious subjects presented Eustachian tube dysfunction. In the Kaplan-Meier curves for levels of consciousness (see Fig. 3) we observed that subjects with a low level of consciousness (RSS score 4-6) presented a higher number of pathological curves, and they appeared sooner than in those subjects with clear consciousness (RSS score 1-3). A Cox regression analysis revealed a statistically significant association between unconsciousness and pathological curve (odds ratio 2.42, 95\% CI 1.65-3.55). Neurologically impaired patients or patients having consciousness disturbance might present poor swallowing and neuromuscular functions. These can lead to Eustachian tube dysfunction and inadequate aeration of the middle ear. ${ }^{12}$
We found no acute otitis media, intracranial infection, or long-term disabilities in critically ill subjects in this study. However, in terms of quality of life for the patient, MEE might cause conductive hearing loss, and it will aggravate preexisting sensorineural hearing loss caused by age (we have to remember that the mean age in our study was 58 years). Therefore, MEE limits the patient's ability to understand commands or explanations, which can impair rehabilitation of the patients. Apart from that, MEE can cause aural fullness and disturbance for the patient.

Among the limitations of this study are the missing observations due to clotted blood, cerumen impactation, or fluid drainage on external ear canal; patient position; cervical collars and head dressings; and lack of patient cooperation. As we have described in the results, the statistical power decreased from a theoretical $80 \%$ to a real $65 \%$ due to missing observations. This fact did not affect the conclusions of this study, because it detected, with statistically significant results, the effect of 18 French NGT on tympanometry curves of ICU patients. Another limitation imposed by the study design was the exclusion criteria for patients with a past history of ear diseases. In this group of patients, who had a basal imbalance of middle ear function, the effects of analyzed predisposing factors to MEE might adversely affect their outcomes to a greater extent. Further studies are required that will take into account patients who have a previous otological pathology.

\section{Conclusions}

In summary, NGTs of 18 French have been demonstrated as an easily modifiable predisposing factor for MEE in ICU patients. In the same way, orotracheal intubation and unconsciousness have been confirmed as risk factors for MEE. In view of previous results, we recommend using the smallest possible diameter of NGT in order to prevent or to minimize the effects of MEE.

\section{REFERENCES}

1. Hamill-Ruth RJ, Ruth RA, Googer K, Volles D, Deivert M, Turrentine B. Use of otoacoustic emissions to screen for hearing loss in critically ill patients. Audiology 1998;37(6):344-352.

2. Hamill-Ruth RJ, Ruth RA. Evaluation of audiologic impairment in critically ill patients: results of a screening protocol. Crit Care Med 2003;31(9):2271-2277.

3. Roth Y, Sokolov M, Adler M, Ezry T, Harell M. Otorhinolaryngological problems occurring within the intensive care unit. Intensive Care Med 2003;29(6):884-889.

4. Halpern NA, Pastores SM, Price JB, Alicea M. Hearing loss in critical care: an unappreciated phenomenon. Crit Care Med 1999; 27(1):211-219.

5. Cavaliere F, Masieri S, Liberini L, Proietti R, Magalini SI. Tympanometry for middle-ear effusion in unconscious ICU patients. Eur J Anaesthesiol 1992;9(1):71-75. 


\section{Middle Ear Effusion in Mechanically Ventilated Patients}

6. Christensen L, Schaffer S, Ross SE. Otitis media in adult trauma patients: incidence and clinical significance. J Trauma 1991;31(11): 1543-1545.

7. Magnano M, Albera R, Lacilla M, Gabini A, Naddeo M, Bruno D. Impedance measurement as a noninvasive technique for the monitoring of intracranial pressure variations. Audiology 1994;33(4): 237-243.

8. Jerger J. Clinical experience with impedance audiometry. Arch Otolaryngol 1970;92(4):311-324.

9. Chung HK, Lin CC, Wang CY, Lin CD, Tsai MH, Chang CS. Improvement in otitis media with effusion in patients undergoing tracheostomy after prolonged endotracheal intubation. J Otolaryngol Head Neck Surg 2009;38(5):532-536.

10. Lin CC, Lin CD, Cheng YK, Tsai MH, Chang CS. Middle ear effusion in intensive care unit patients with prolonged endotracheal intubation. Am J Otolaryngol 2006;27(2):109-111.

11. Cavaliere F, Masieri S, Proietti R, Magalini SI. pH and electrolytes in nasal secretum of intensive care unit patients. Resuscitation 1988; 16(2):133-137.

12. Vento BA, Durrant JD, Palmer CV, Smith EK. Middle ear effects secondary to nasogastric intubation. Am J Otol 1995;16(6): 820-822. 